\title{
MINDFULNESS Y TÉCNICAS DE ESTUDIO EN UN CASO DE BAJO RENDIMIENTO ACADÉMICO
}

\author{
Laura del Carmen Sánchez-Sánchez \\ Departamento de Psicología Evolutiva y de la Educación \\ Universidad de Granada \\ Icsanchezsa@ugr.es \\ Teresa María Perandones González \\ Departamento de Psicología Evolutiva y de la Educación \\ Universidad de Granada \\ tmperandones@ugr.es
}

Fecha de Recepción: 8 Enero 2019

Fecha de Admisión: 30 Abril 2019

\section{RESUMEN}

El objetivo de este estudio de caso único fue aplicar Mindfulness combinado con técnicas de estudio para mejorar la concentración del alumno, así como su estado de ánimo y afrontamiento de su vida académica. El participante tiene 13 años y acude a consulta porque ha suspendido 6 asignaturas de $2^{\circ}$ de ESO y presenta depresión grave y ansiedad leve, así como un patrón de evitación de todo lo académico. Respecto a la metodología, se trata de un diseño A-B-A, donde se aplicaron los cuestionarios de ansiedad y depresión de Hamilton validadas en castellano y la Adaptación Española del Cuestionario de Aceptación y Acción. Asimismo, completó una serie de autorregistros durante el tratamiento: por una parte, tiempo que dedicaba a estudiar y a hacer trabajos, si practicaba 0 no el ejercicio de Mindfulness recomendado, aprovechamiento de esas horas de estudio, horas de sueño y tiempos de descanso; por otra parte, intensidad de sus preocupaciones, intensidad con que se dirige a sus valores y satisfacción con ese día. Se aplicó el protocolo de la Meditación Fluir y, posteriormente, se trabajaron técnicas de estudio desde la perspectiva de la conciencia plena. El alumno mejoró sus puntuaciones en depresión, ansiedad y AAQ de manera clínicamente significativa y mejoró su rendimiento académico, llegando a aprobar todas las asignaturas suspendidas. Como conclusión general, el Mindfulness, como estrategia metacognitiva, combinada después con técnicas de estudio, puede ser una herramienta útil, no sólo para mejorar la concentración, sino también para sobrellevar las tareas académicas de una manera más positiva.

Palabras clave: rendimiento académico; problemas socioemocionales; Mindfulness; técnicas de estudio; metacognición 


\title{
MINDFULNESS Y TÉCNICAS DE ESTUDIO EN UN CASO DE BAJO RENDIMIENTO ACADÉMICO
}

\begin{abstract}
Mindfulness and study skills in a case of low academic performance. The objective of this case study was the application of Mindfulness combined with study skills in order to improve the student concentration, mood and coping mechanism with his academic life. The participant is a 13years-old secondary student and comes to therapy because he has failed 6 subjects and show serious depression, mild anxiety and experiential avoidance pattern with respect to the academic field. Regarding the methodology, it is an A-B-A design was carried out, in which Hamilton's questionnaires of anxiety and depression and Acceptation and Action Questionnaire were applied. The participant also fulfilled some self-records along the treatment: on the one hand, time studying and doing homework, practice of Mindfulness task, academic progress, time of sleep and breaks; on the other, intensity of worries, intensity to go towards values and satisfaction with that day. FlowMeditation and study skills from a Mindfulness perspective were applied. The student improved his depression, anxiety and $A A Q$ scores in a clinically significant way and make progress in his academic performance, passing all the failed subjects. As a general conclusion, Mindfulness, as a metacognitive method, combined with study skills, can be a useful tool, not only to improve concentration, but also, to cope with academic tasks in a more positive way.
\end{abstract}

Keywords: academic performance; socioemotional problems; Mindfulness; study skills; metacognition

\section{INTRODUCCIÓN}

El alumnado frecuentemente presenta problemas para concentrarse cuando intenta estudiar 0 realizar tareas escolares (Fernández y Ponte, 2013). Esta situación puede repercutir en su rendimiento y en la satisfacción con su vida académica, llegando incluso en ocasiones a influir sobremanera en su estado de ánimo, por cuanto su manera de afrontar la vida académica adopta patrones poco adaptativos, por ejemplo, patrones de evitación.

A menudo las técnicas de estudio son insuficientes por sí solas para mejorar el rendimiento del alumnado y su manera de enfrentarse a los estudios (Sobrado, Cauce y Rial, 2002). El punto de partida de este estudio es, por tanto, aportar una herramienta metacognitiva, en este caso Mindfulness, que ayude al alumnado a mejorar su concentración y la manera de afrontar su vida académica. Ésta se podría enmarcar dentro de las estrategias para "aprender a aprender" (Alonso, Gallego y Honey, 1997), lo que las diferencia de otras técnicas de estudios que se centran simplemente en aprender y no en "aprender a aprender". Por otra parte, no podemos olvidar que Mindfulness se encuadra dentro de las "Terapias de Tercera Generación" (Hayes, 2004). Estas terapias no van dirigidas a la modificación del síntoma (sea ansiedad, depresión, etc.), sino a que la persona actúe con responsabilidad respecto a las elecciones que haga en su vida y acepte los eventos internos (emociones, pensamientos, sensaciones corporales, etc.) que aparezcan durante ese camino elegido. Esto choca frontalmente con otras terapias psicológicas o incluso con lo que se espera socialmente de una persona en determinadas circunstancias. Por ejemplo, si alguien está triste, se le dice "alégrate", "diviértete"; si alguien está nerviosos, se le indica "relájate"; 0 si tiene algún pensamiento que le atormenta, le apuntamos "no pienses más en ello". Lo que en ocasiones se nos escapa es que, si esa persona pudiera hacer todo esto que se le indica, ya lo habría hecho motu proprio sin solicitar ningún tipo de ayuda. Además, estas indicaciones promueven un patrón de evitación de los eventos internos, un hecho que, frecuentemente, no sólo no se consigue (véase una revisión de las investigaciones sobre el efecto rebote del contenido suprimido en Rassin, Merckelbach y Muris, 2000), sino que mientras se involucrna en evitar todo ello acaba yendo en contra de lo que valoran en su vida. 
Mindfulness es una traducción de la palabra Sati, de la lengua Pali, sin transcripción directa al castellano, aunque se suele traducir como atención y conciencia plena (García y Demarzo, 2015). Mindfulness se puede definir como aquella atención que no juzga, sino que observa con ecuanimidad todo lo que ocurre en el presente, cada conducta, pensamiento, sentimiento o sensación se explora y acepta tal como es, sin evitar ningún evento interno que surja en ese momento (Bishop et al., 2004).

Puesto que Mindfulness mejora varios aspectos cognitivos (atención, memoria, etc.) y socioemocionales (autoestima, autoconcepto, ansiedad, depresión, etc.), se ha aplicado en números ocasiones a contextos educativos. Concretamente, para el tema que nos ocupa, ha arrojado prometedores resultados en estudiantes de secundaria (Bei et al. 2013; Franco, Mañas, Cangas, Gallego, 2011; Huppert and Johnson, 2010; Raes, Griffith, Van der Gucht y Williams, 2014).

Sin embargo, también se ha puntualizado que, aunque la aplicación de Mindfulness puede ser útil para mejor aspectos cognitivos y socioemocionales del alumnado, obtiene pobres resultados en cuanto a la mejora de las conductas académicas (Maynard, Solis, Miller y Brendel, 2017). De todo lo anterior se desprende que la combinación de ambas herramientas, técnicas de estudio y Mindfulness, puede ser lo más acertado en estos contextos y no sólo una de ellas.

Por tanto, el principal objetivo de este estudio de caso único fue aplicar Mindfulness combinado con técnicas de estudio para mejorar la atención del alumno, así como su estado de ánimo y afrontamiento de su vida académica.

\section{MÉTODO}

\section{Diseño}

Se trata de un estudio con diseño A-B-A, donde se aplicaron una serie de instrumentos de evaluación antes y después del tratamiento combinado de Meditación Fluir y técnicas de estudio, así como en el seguimiento de un mes.

\section{Participante}

El participante es un chico de 13 años que acude a consulta porque ha suspendido 6 asignaturas de $2^{0}$ de ESO. Señala que le va peor desde que sus padres lo cambiaron a un centro bilingüe, donde lo encuentra todo más difícil y además no está con el grupo de amigos de su antiguo colegio. Todo ello le ha llevado a tener dificultades para concentrarse en las asignaturas, a pesar de que indica que le dedica muchas horas al día, y para desarrollar las tareas encomendadas. Además, desde que está en este centro y ha empeorado su rendimiento, también está más triste y ansioso. Ha llegado a desarrollar un patrón de evitación de numerosas actividades: estudiar, hacer las actividades, organizarse, tareas en el hogar relacionadas con prepararse para ir al centro de enseñanza (como levantarse solo, hacerse el desayuno, prepararse la mochila, etc.) e incluso ir a clase. Este patrón de evitación está repercutiendo no sólo en su rendimiento académico, que ha empeorado, sino también en la asunción de responsabilidades en el hogar, en su satisfacción vital, estado de ánimo, etc. Esto se conoce en Terapias de Tercera Generación como Evitación Experiencial y puede ser clínicamente significativa en la medida en que afecte a sus valores vitales. Además de presentar un patrón de Evitación Experiencial, manifestaba también depresión grave y ansiedad leve antes de comenzar la terapia, según sus puntuaciones en los instrumentos utilizados. En la línea de base previa la intensidad de sus preocupaciones era alta $(>5)$ y tanto la intensidad con la que actuaba en dirección a sus valores como su satisfacción vital era bajas $(<5)$. 


\section{Instrumentos}

Los instrumentos aplicados fueron: Ios cuestionarios de ansiedad y depresión de Hamilton validadas en castellano (Lobo, et al., 2002; Ramos-Brieva, 1986), la Adaptación Española del Cuestionario de Aceptación y Acción (AAQ, Barraca-Mairal, 2004) y autorregistros para casa.

La escala de Hamilton para la ansiedad (HAM-A) en su validación en castellano (Lobo, et al., 2002) está compuesta por 14 ítems que exploran diversos estados de tensión, ansiedad, síntomas neurovegetativos y somáticos. Se evalúa con una escala tipo Likert que va desde 0 (ausente) a 4 (muy grave/incapacitante). La ansiedad se considera leve si oscila entre 18 y 25 puntos, moderada, entre 25 y 30 y severa si es mayor de 30 . La versión española de la escala muestra una adecuada consistencia interna ( = .89).

La escala depresión de Hamilton validadas en castellano (Ramos-Brieva, 1986) está compuesta por 17 ítems evaluados con una escala tipo Likert que oscila desde 0 (ausente) hasta 4 , graduando el nivel de afectación en cada uno de ellos. Los valores entre 0 y 7 indican que no hay depresión, entre 8 y 13 ligera, entre 14 y 18 moderada, entre 19 y 22 grave y más de 23 muy grave. La escala muestra una adecuada consistencia interna $(=.7)$.

La Adaptación Española del Cuestionario de Aceptación y Acción (AAQ, Barraca-Mairal, 2004) es un cuestionario que evalúa la evitación experiencial y la aceptación psicológica, dos conceptos claves en las Terapias de Tercera Generación. Está compuesto por 9 ítems en los que el participante ha de evaluar en qué medida son aplicables a sí mismo con una escala tipo Likert que va desde 1 (nunca verdad) a 7 (siempre verdad). Una mayor puntuación indica un mayor grado de evitación experiencial, mientras que una puntuación menor indica un mayor grado de aceptación psicológica. En muestras clínicas la media en esta escala fue de 44.71 (SD= 8.42). La versión española de la escala muestra adecuadas propiedades psicométricas, mostrando una elevada su consistencia interna $(=.74)$.

Así mismo, el alumno debía completar una serie de autorregistros durante el tratamiento, donde anotaba diariamente: por una parte, las horas que dedicaba a estudiar y a hacer trabajos, si practicaba o no el ejercicio de Mindfulness recomendado, el aprovechamiento de esas horas de estudio, las horas de sueño y los tiempos de descanso; por otra parte, la intensidad de sus preocupaciones, la intensidad con que se dirige a sus valores y la satisfacción con ese día. Estas tres últimas medidas oscilaban de 1 a 10.

\section{Procedimiento}

Se partió de un abordaje holístico del problema (Micollini, 2006), atendiendo tanto a las condiciones internas (descanso, alimentación, afrontamiento del estrés, tener metas y valores de partida, etc.) para el estudio, como a las externas o ambientales (iluminación, ruido, etc.) que también repercuten en él.

Respecto a la intervención, se aplicó el protocolo de Meditación Fluir (Franco, 2009), que combina los fundamentos de la Terapia de Aceptación y Compromiso (ACT), para ubicar al paciente en la filosofía de las Terapias de Tercera Generación (Hayes, 2004) y Mindfulness durante los dos primeros meses. El componente de ACT se utilizó especialmente para clarificar los valores que eran importantes para el alumno y que serían los que le ayudarían a ver el camino a seguir y en qué medida se estaba o no desviando de él. Se aplicó la Meditación Fluir en primer lugar, para dotar al alumno de la herramienta que le permitiría concentrarse con mayor facilidad en todas las actividades relacionadas con su vida académica: planificación del tiempo, elección del entorno de estudio, rendimiento de las horas dedicadas a ello, etc.

Posteriormente, se trabajaron técnicas de estudio que, si bien el alumno ya conocía parcialmente, se abordaron desde esta nueva perspectiva de la conciencia plena. En primer lugar, se tra- 
bajó en todas aquellas variables que el alumno nos indicó que podrían estar afectando su rendimiento: elección de un entorno de estudio ruidoso y con poca luz, estar rodeado de numerosos distractores (decoración excesiva, acceso constante al móvil, etc.), alimentación poco saludable, pocas horas de sueño, escasa planificación de las horas de estudio y de descanso, etc. Posteriormente, las técnicas empleadas fueron aquellas que se utilizan el método de estudio EPLERR: Explorar, Preguntar, Leer, Esquematizar, Recitar y Resumir (Hernández, 1988). Si bien, se atendió a los estilos de aprendizaje particulares de este alumno, así como a sus preferencias. Por ejemplo, en el apartado de Esquematizar, dado que al alumno le gustaba dibujar y lo hacía con destreza, había desarrollado una buena memoria visual. Así, le resultaba más útil hacer esquemas que resúmenes, porque a golpe de vista podía recordar todo lo esquematizado. Este hecho se tuvo en cuenta en la aplicación del método, siendo los valores y los estilos de aprendizaje del alumno los que guiaron la intervención. La indicación sugerida al alumno era que, al llevar a cabo cada técnica del método EPLERR, lo hiciera con atención plena. Si estaba en la parte de la lectura, por ejemplo, y la mente se le iba a cualquier otro lugar que no fuera lo que estaba leyendo, ya sabía que tenía que darse cuenta de ello y volver una y otra vez a la lectura, igual que ya hacía con el ejercicio de respiración que practicaba.

\section{Análisis de datos}

Los datos que se muestran a continuación son meramente descriptivos, puesto que se trata de un estudio de caso único exploratorio.

\section{RESULTADOS}

El alumno mejoró sus puntuaciones en depresión y ansiedad de manera clínicamente significativa, así como en el cuestionario de AAQ. En depresión, bajó de "depresión grave" a ausencia de la misma. En el AAQ también dejó de presentar un patrón de Evitación Experiencial (véase Tabla 1).

Tabla 1

Puntuaciones del alumno en las escalas de ansiedad y depresión de Hamilton y en el $A A Q$ en el pre-test, post-test y al mes de seguimiento

\begin{tabular}{lccc}
\hline & \multicolumn{2}{c}{ Estado De Ánimo } & Evitación Experiencial \\
\cline { 2 - 4 } & $\begin{array}{l}\text { Ansiedad } \\
\text { (HAM-A) }\end{array}$ & $\begin{array}{c}\text { Depresión } \\
\text { (HAM-D) }\end{array}$ & AAQ \\
\hline Ev. LB & 19 & 20 & 50 \\
Ev. Post & 17 & 6 & 38 \\
Seguimiento al mes & 9 & 2 & 35 \\
\hline
\end{tabular}

Por otra parte, fue aumentando progresivamente no sólo las horas sueño y de estudio, sino también el aprovechamiento de este tiempo. El aumento en las puntuaciones corrió paralelo a la prácti- 


\section{MINDFULNESS Y TÉCNICAS DE ESTUDIO EN UN CASO DE BAJO RENDIMIENTO ACADÉMICO}

ca del ejercicio de Mindfulness en casa y el hecho de comenzar a actuar en dirección a sus valores más a menudo. En la siguiente figura podemos observar cómo fue incrementando progresivamente las acciones en dirección a sus valores y cómo esto aumentó también su satisfacción vital, a pesar de que algunos días la intensidad de las preocupaciones continuaba siendo elevada (véase Figura 1). Los primeros 7 días son los de línea base, los 7 siguientes una evaluación en mitad del tratamiento, los siguientes en el post-tratamiento y, por último, al mes de seguimiento.

Figura 1. Evolución en semanas de la Intensidad de las preocupaciones, la Intensidad de sus actos en dirección a valores y el Grado de satisfacción con ese día.

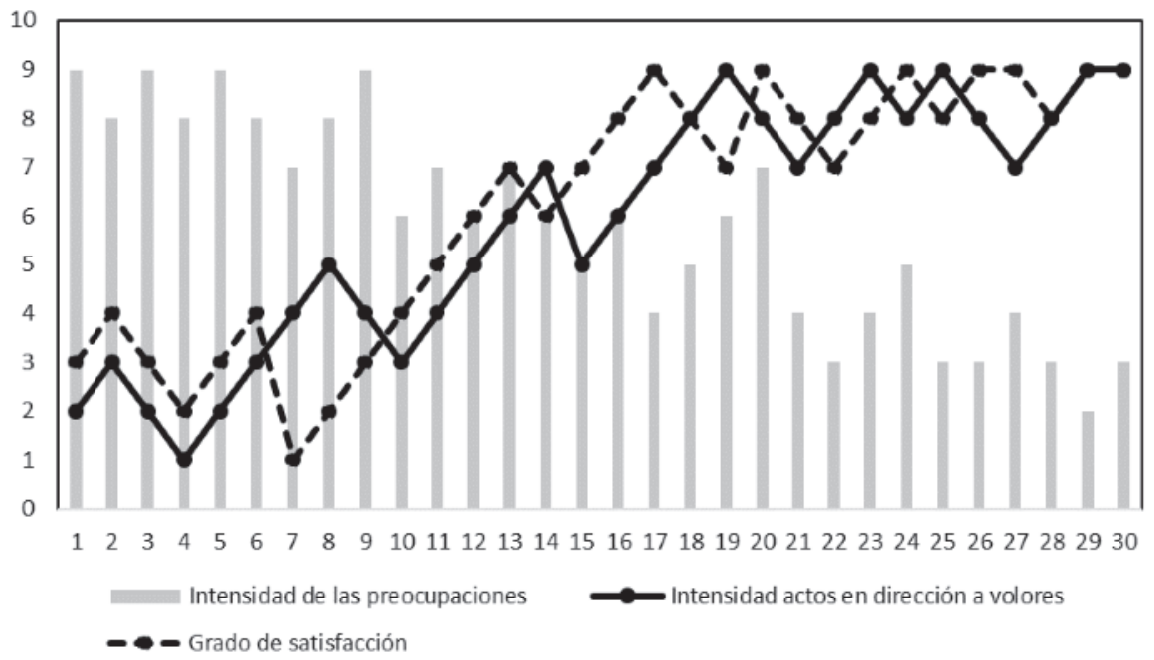

Todo ello supuso una mejora de su rendimiento académico, llegando a aprobar todas las asignaturas suspendidas al final del curso. Además, el propio alumno cuenta en sesión que no sólo ha mejorado su rendimiento académico, sino que ahora se concentra mejor también en el resto de áreas de su vida, se planifica mejor, asume más responsabilidades, etc. Así mismo, los padres, que acudieron en las primeras y en las últimas sesiones, corroboran sus avances.

\section{DISCUSIÓN}

El rendimiento académico está relacionado con los hábitos de estudio (Fullana, 2008; Meneghetti et al., 2007; Nonis y Hudson, 2010). No obstante, en este paciente y en muchos otros estudiantes, las técnicas de estudio por sí solas no son suficientes para asegurar que el alumnado esté concentrado en ellas mientras las lleva a cabo. Además, el fracaso escolar había repercutido en su estado de ánimo, presentando depresión grave cuando acudió a consulta, y habiendo desarrollado un patrón de Evitación Experiencial que estaba afectando a sus valores vitales.

Según muestran los resultados del $A A Q$, tras la intervención, su patrón de Evitación Experiencial mejoró, mostrando más aceptación psicológica. Éste es un objetivo crucial de las Terapias de Tercera Generación (Wilson y Luciano, 2014). Los registros semanales también revelaron la ruptura de este patrón, puesto que, a pesar de la intensidad de sus preocupaciones, continuó actuando con intensidad en dirección a sus valores. Este hecho supuso también un incremento de la satisfacción vital, que corrió paralela al aumento de sus actos. Por otra parte, y aunque éste no suele ser 
un objetivo prioritario de las Terapias de Tercera Generación por el marco teórico del que parten, en el post-test y el seguimiento también disminuyó la intensidad de sus preocupaciones, la depresión y la ansiedad. Esto concuerda con otros estudios previos donde se han evaluado este tipo de variables (Franco, Mañas, Cangas, Gallego, 2011; Raes, Griffith, Van der Gucht y Williams, 2014).

Aunque el cambio en el ambiente de estudio y la aplicación del método de estudio EPLERR haya podido influir en los resultados, es probable que el efecto de las técnicas de estudio se haya visto potenciada por la aplicación de Mindfulness durante su uso, ya que muchas de ellas las conocía antes de la aplicación de Mindfulness y aun así no estaba obteniendo con ellas el rendimiento académico deseado. Por otra parte, es probable que la administración de Mindfulness por sí sola tampoco hubiera conseguido los resultados deseados, como apuntan algunos autores (Maynard, Solis, Miller y Brendel, 2017), puesto que carecía de hábitos de estudio como tales cuando acudió a consulta.

Por último, es preciso destacar no sólo los datos cuantitativos, sino la relevancia clínica de los resultados. Los objetivos planteados al inicio se cumplieron, mejorando el rendimiento académico del alumno, pero también su satisfacción vital. Esto ocurrió no sólo en el ámbito académico, sino también en la mejora de sus relaciones familiares, de amistad e incluso de ocio; dado que, al planificar mejor su tiempo, tenía más momentos de descanso y, al igual que los de estudio, estos también se vivían con mayor atención plena.

\section{CONCLUSIONES}

Las técnicas de estudio por sí solas pueden ser insuficientes para ayudar al alumnado a concentrarse en las tareas académicas que le son encomendadas. El hecho de dedicar un número de horas determinadas al estudio o a la realización de tareas no asegura su aprovechamiento. El fracaso escolar puede repercutir también en el estado de ánimo de los estudiantes y, sobre todo, en su satisfacción vital, puesto que su vida académica ocupa mucho tiempo diario. El hecho de incluir Terapias de Tercera Generación, especialmente Mindfulness, como estrategia metacognitiva en los centros de enseñanza reglada y combinarla después con técnicas de estudio, puede ser una herramienta útil, no sólo para ayudar a concentrarse mejor, sino también para sobrellevar las tareas académicas de una manera más positiva.

Serán necesarias futuras investigaciones donde se salven algunas de las limitaciones que presentan los estudios precedentes en los que se ha aplicado Mindfulness en un contexto educativo, como ya han señalado algunos autores (Felver, Celis-de Hoyos, Tezanos y Singh, 2016). Es preciso llevar a cabo estudios de este tipo con muestras amplias, donde el grupo control no sea sólo una condición de lista de espera, sino un grupo activo. La aplicación de técnicas de estudio en solitario en el grupo control y de Mindfulness más técnicas de estudio en el experimental puede ayudar a dilucidar qué tipo de intervención puede ser más eficaz en la mejora del rendimiento académico del alumnado, así como en otras variables socioemocionales y, sobre todo, en la satisfacción académica y vital de los estudiantes.

\section{REFERENCIAS BIBLIOGRÁFICAS}

Alonso, C. M., Gallego, D. J. y Honey, P. (1997). Los estilos de aprendizaje: procedimientos de diagnóstico y mejora. Mensajero.

Barraca-Mairal, J. (2004). Spanish adaptation of the Acceptance and Action Questionnaire (AAQ). International Journal of Psychology and Psychological Therapy, 4(3), 505-516.

Bei, B., Byrne, M. L., Ivens, C., Waloszek, J., Woods, M. J., Dudgeon, P., y Allen, N. B. (2013). Pilot study of a mindfulness based, multicomponent, in school group sleep intervention in adolescent girls. Early Intervention in Psychiatry, 7, 213-220. 


\section{MINDFULNESS Y TÉCNICAS DE ESTUDIO EN UN CASO DE BAJO RENDIMIENTO ACADÉMICO}

Fernández, M. M. y Ponte, L. G. (2013). Cómo mejorar la autorregulación del estudio en la Educación Secundaria. Valoración de un programa de acción tutorial. Contextos Educativos. Revista de Educación, (5), 215-226.

Felver, J. C., Celis-de Hoyos, C. E., Tezanos, K., y Singh, N. N. (2016). A systematic review of mindfulness-based interventions for youth in school settings. Mindfulness, 7(1), 34-45.

Franco, C. (2009). Meditación Fluir Para Serenar el Cuerpo y la Mente. Madrid: Bubok.

Franco, C., Mañas, I., Cangas, A. J., y Gallego, J. (2011). Exploring the effects of a mindfulness program for students of secondary school. International Journal of Knowledge Society Research (IJKSR), 2(1), 14-28.

Fullana, J. (2008). La investigació sobre l'exit i el fracàs escolar des de la perspectiva dels factors de risc. Implicacions per a la recerca i la pràctica, tesis doctoral, Departament de Pedagogia. Universitat de Girona.

Hayes, S. C. (2004). Acceptance and commitment therapy, relational frame theory, and the third wave of behavioral and cognitive therapies. Behavior therapy, 35(4), 639-665.

Hernández, F. (1988). Métodos y técnicas de estudio. Universidad de Colombia, Bogotá.

Huppert, F. A., y Johnson, D. M. (2010). A controlled trial of mindfulness training in schools: the importance of practice for an impact on well-being. The Journal of Positive Psychology, 5, 264-274.

Lobo, A., Chamorro, L., Luque, A., Dal-Ré, R., Badia, X., Baró, E., y Grupo de Validación en Español de Escalas Psicométricas (GVEEP. (2002). Validación de las versiones en español de la Montgomery-Asberg Depression Rating Scale y la Hamilton Anxiety Rating Scale para la evaluación de la depresión y de la ansiedad. Medicina clínica, 118(13), 493-499.

Maynard, B. R., Solis, M. R., Miller, V. L. y Brendel, K. E. (2017). Mindfulness-Based Interventions for Improving Cognition, Academic Achievement, Behavior, and Socioemotional Functioning of Primary and Secondary School Students. Campbell Systematic Reviews 2017: 5. Campbell Collaboration

Meneghetti, C., De Beni, R., \& Cornoldi, C. (2007). Strategic knowledge and consistency in students with good and poor study skills. European Journal of Cognitive Psychology, 19(4-5), 628-649.

Micollini, A. (2006). Competencias para un estudio eficaz. Córdoba: Editorial Brujas.

Nonis, S. A., \& Hudson, G. I. (2010). Performance of college students: Impact of study time and study habits. Journal of education for Business, 85(4), 229-238.

Raes, F., Griffith, J. W., Van der Gucht, K., y Williams, J. M. G. (2014). School-based prevention and reduction of depression in adolescents: A cluster-randomized controlled trial of a mindfulness group program. Mindfulness, 5(5), 477-486.

Ramos-Brieva, J. A. (1986). Validación de la versión castellana de la escala de Hamilton para la depresión. Actas Luso Esp. Neurol. Psiquiatr., 14, 324-334.

Rassin, E., Merckelbach, H., \& Muris, P. (2000). Paradoxical and less paradoxical effects of thought suppression: A critical review. Clinical Psychology Review, 20(8), 973-995.

Sobrado Fernández, L. M., Cauce Santalla, A. y Rial Sánchez, R. (2002). Las habilidades de aprendizaje y estudio en la educación secundaria: estrategias orientadoras de mejora.

Wilson, K. G., y Soriano, M. C. L. (2014). Terapia de aceptación y compromiso (ACT). Madrid: Ediciones Pirámide. 